\title{
Otimização por Page do processo de \\ secagem em leito de espuma para produção de café solúvel
}

\section{Page optimization of the foam-mat drying process to soluble coffee production}

\author{
Gustavo Henrique Souza Silva ${ }^{1}$, Ana Paula Pereira Bressani², \\ Mateus da Silva Junqueira ${ }^{1 *}$ (D)
}

${ }^{1}$ Universidade Federal de São João Del-Rei (UFSJ), Departamento de Engenharia de Alimentos, Sete Lagoas/MG Brasil

${ }^{2}$ Universidade Federal de Lavras (UFLA), Departamento Ciência dos Alimentos, Lavras/MG - Brasil

*Corresponding Author: Mateus da Silva Junqueira, Universidade Federal de São João Del-Rei (UFSJ), Departamento de Engenharia de Alimentos, Rua Sétimo Moreira Martins, 188, Itapuã, Caixa Postal: 56 CEP: 35702-031, Sete Lagoas/MG - Brasil, e-mail: mateusjunqueira@ufsj.edu.br

Cite as: Silva, G. H. S., Bressani, A. P. P., \& Junqueira, M. S. (2020). Page optimization of the foam-mat drying process to soluble coffee production. Brazilian Journal of Food Technology, 23, e2019145.

https://doi.org/10.1590/1981-6723.14519

\begin{abstract}
Resumo
A expansão da produção de café solúvel é uma alternativa ao consumo do café tradicional, tornando-se necessária para atender às novas exigências dos consumidores que buscam conveniência e praticidade. Diante desse cenário, o estudo de técnicas que simplificam e reduzem custos para a obtenção de café solúvel se torna mais pertinente. Portanto, o objetivo deste trabalho foi otimizar o processo de secagem de extrato de café em leito de espuma, por meio do ajuste ao Modelo de Page por regressão não linear. Foram avaliados dois métodos para a concentração do extrato: (A) em evaporador rotativo e (B) em recipiente de inox aberto. A cinética de secagem do extrato de café em leito de espuma sob as temperaturas de $40,50,60,70$ e $80^{\circ} \mathrm{C}$ foi descrita pelo modelo. Além disso, foi proposta uma equação multivariada para a secagem mediante deteç̧ão da influência da temperatura no parâmetro $k$, do Modelo de Page, e posterior validação dos resultados. $O$ tempo para obtenção do extrato concentrado foi próximo para os dois métodos de concnetração (A: 2 horas e 30 minutos; B: 2 horas e 40 minutos). $O$ modelo de Page apresentou ótimo ajuste com valores de coeficientes de determinação acima de $99 \%$. Por meio dos resultados obtidos, foi confirmado que a temperatura é um fator determinante na velocidade de perda de umidade do produto, apresentando tempos de secagem menores com o aumento da temperatura. A correlação entre os dados experimentais e preditos foi satisfatória para validar os resultados. O modelo matemático também pode auxiliar na correlação dos parâmetros de secagem com alterações físico-químicas e sensoriais do café solúvel, a fim de melhorar os parâmetros tecnológicos.
\end{abstract}

Palavras-chave: Cinética de secagem; Modelo de Page; Leito fluidizado; Extrato de café; Temperaturas de secagem; Modelo matemático. 


\begin{abstract}
The expansion of the soluble coffee production is an alternative to the traditional coffee consumption, which is necessary to meet the new demands of consumers looking for convenience and practicality. Thus, this study of techniques that simplify and reduce costs to obtain soluble coffee become even more pertinent. Therefore, the objective of this work was to optimize the drying process of coffee extract in the foam bed by adjusting the Page Model by non-linear regression. Two methods for the concentration of the extract were evaluated: $(A)$ in rotary evaporator and (B) in open stainless-steel container. The kinetics of drying the coffee extract in a foam bed under the temperatures of $40,50,60,70$ and $80^{\circ} \mathrm{C}$ were described by the model. Besides that, a multivariate equation was proposed for drying by detecting influence of temperature on the $\mathrm{k}$ parameter, of the Page Model and later validation of the results. The time to obtain the concentrated extract was close to the two concentration methods (A- 2 hours and 30 minutes and B- 2 hours and 40 minutes). The Page model presented a good fit with values of determination coefficients above $99 \%$. The results confirmed that the temperature is a determinant factor in the product moisture loss speed, presenting shorter drying times with the temperature increase. The correlation between experimental and predicted data was satisfactory for the validation of the results. The mathematical model can also assist in the correlation of drying parameters with physicochemical and sensory changes of soluble coffee in order to improve technological parameters.
\end{abstract}

Keywords: Drying kinetics; Page model; Fluidized bed; Coffee extract; Temperatures of drying; Mathematical model.

\title{
1 Introdução
}

O café é uma bebida popular e amplamente consumida por sua composição e propriedades estimulantes (Evangelista et al., 2014; Martinez et al., 2017). O Brasil é o maior produtor e exportador global de café, com quase 62 milhões de sacas beneficiadas em 2018 (International Coffee Organization, 2019), sendo Minas Gerais, Espírito Santo e São Paulo os principais estados produtores (Companhia Nacional de Abastecimento, 2018).

Segundo Esquivel \& Jiménez (2012), cerca de 50\% da produção mundial de café verde foi destinada às indústrias de café instantâneo. Atualmente, no Brasil, durante o período de junho de 2018 a janeiro de 2019, houve um aumento de 12,9\% no volume de café solúvel exportado (Conselho dos Exportadores de Café do Brasil, 2019). Esse aumento nas exportações pode ser devido, principalmente, à facilidade e à rapidez no preparo, que são características muito procuradas pelos consumidores (Cruz et al., 2017).

O café solúvel ou instantâneo refere-se ao café em pó ou em grânulo, produzido a partir da desidratação do extrato aquoso do café (Coffea arabica e/ou outras espécies do gênero Coffea) torrado e moído (Cruz et al., 2017; Santos et al., 2018).

A secagem em leito de espuma é uma técnica em que um concentrado líquido adicionado de um agente espumante adequado é submetido à desidratação (Araújo et al., 2017) a uma temperatura relativamente baixa. É uma alternativa aos métodos convencionais de secagem, por sua simplicidade, relação custo-benefício, secagem em alta velocidade e melhor qualidade do produto (Hardy \& Jideani, 2017). O produto seco por esse método possui propriedades desejadas, como reidratação, densidade controlada e retenção de voláteis que seriam perdidos durante outros métodos de secagem (Kandasamy et al., 2014), característica importante para o café solúvel.

A aplicação de modelos matemáticos e o estudo de curvas de secagem têm sido de grande importância, pois permitem uma maior compreensão do processo de desidratação, otimizando parâmetros como tempo e temperatura, visando ao aumento das qualidades sensorial e tecnológica do produto (Buljat et al., 2019; Franco et al., 2016).

Neste contexto, o objetivo do presente trabalho foi utilizar a técnica em leito de espuma para produzir café solúvel e avaliar a cinética de secagem do extrato por meio do modelo matemático Page. 


\section{Material e métodos}

\subsection{Obtenção dos extratos}

O café moído e o emulsificante comercial Emulstab ${ }^{\circledR}$ (produto à base de monoglicerídeos destilados, monoestearato de sorbitana e polissorbato 60) foram adquiridos no comércio local de Sete Lagoas, em Minas Gerais, Brasil.

O extrato foi obtido por infusão em coador de $60 \mathrm{~g}$ do pó de café em $1.000 \mathrm{~mL}$ de água fervente (seguindo as instruções do fabricante), obtendo-se um volume final de $800 \mathrm{~mL}$, que foi dividido em dois volumes iguais (400 mL), a fim de avaliar dois métodos para a concentração do extrato: (A) em evaporador rotativo Solab, modelo SL126 (simulação de concentração sob vácuo parcial), e (B) em recipiente de inox de $3 \mathrm{~L}$, simulando tacho aberto. O volume final de cada extrato concentrado foi de $100 \mathrm{~mL}$ (Bayindirli, 1993).

Foi adicionado 6\% do agente emulsificante Emulstab ${ }^{\circledR}$ separadamente, em cada extrato concentrado, e misturados em batedeira doméstica Philco modelo Paris (dez minutos na velocidade máxima) para a obtenção das emulsões.

\subsection{Secagem em leito de espuma}

A massa de cada emulsão foi medida e colocada em bandejas quadradas de aço inoxidável $(25 \times 25 \times 10 \mathrm{~cm})$, para a realização da secagem em um protótipo de secador "tipo túnel" com controle de temperatura, velocidade do ar de secagem e módulo de aquisição de dados de massa, umidade e temperatura em tempo real. As temperaturas de secagem utilizadas para a obtenção do café solúvel foram: 40, 50, 60, 70 e $80^{\circ} \mathrm{C}$ (Araújo et al., 2017). A velocidade do ar de secagem foi determinada com termoanemômetro digital modelo TAFR 190 da Instrutherm e fixada em $0,5 \mathrm{~m} / \mathrm{s}$, a fim de evitar arraste de produto desidratado, em razão do baixo peso das partículas obtidas.

Durante o processo de secagem, as massas dos tratamentos foram medidas continuamente em balança Shimadzu e registradas automaticamente a cada 30 segundos, em planilha eletrônica, com software aquisitor de dados (Windows Direct ${ }^{\circledR}$ ), até a estabilização do processo (obtenção de massa constante).

Os dados de massa registrados em tempo real durante o processo de secagem foram convertidos em razão de umidade (RU), conforme a Equação 1:

$\mathrm{RU}=\frac{(\text { massa pesada do produto }- \text { massa de equilíbrio })}{(\text { massa inicial }- \text { massa de equilíbrio })}$

\subsection{Análise estatística}

Os dados obtidos em razão de umidade e tempo foram ajustados ao modelo de Page $\left(R U=e^{(-k t)^{n}}\right)$ por regressão não linear, gerando equações referentes a cada temperatura de secagem. A modelagem matemática foi realizada em software livre de planilha eletrônica.

Além disso, também foi proposta uma equação multivariada que explicasse o processo, correlacionando razão de umidade, temperatura e tempo de secagem. Dessa forma, procedeu-se à nova regressão não linear por meio do modelo de Page, sendo o parâmetro $\mathrm{k}$ descrito linearmente em função da temperatura de secagem. Os parâmetros do modelo também foram submetidos ao teste estatístico t de Student a nível de 5\% de significância para verificar o ajuste (Roncheti, 2014).

Após o ajuste das curvas de secagem ao modelo de Page, o processo de obtenção do café solúvel foi reproduzido para a validação dos resultados. Os dados obtidos nesse segundo experimento foram aplicados tanto às equações para cada curva de temperatura de secagem quanto à equação multivariada, geradas pelo primeiro experimento. Os erros obtidos pela dispersão entre os valores experimentais e preditos pelo modelo 
foram calculados por meio de indicadores estatísticos (erro absoluto médio (Equação 2), erro médio de estimativa (Equação 3), raiz do erro quadrático médio (Equação 4) e coeficiente de Willmott (Equação 5)) para verificar a validade dos resultados.

$$
\begin{aligned}
& \operatorname{EAM}=\frac{\sum_{\mathrm{i}=1}^{\mathrm{n}}|\mathrm{Pi}-\mathrm{Oi}|}{\mathrm{n}} \\
& \operatorname{EME}=\frac{\sum_{\mathrm{i}=1}^{\mathrm{n}}(\mathrm{Pi}-\mathrm{Oi})}{\mathrm{n}} \\
& \mathrm{REQM}=\sqrt{\frac{\sum_{\mathrm{i}=1}^{\mathrm{n}}(\mathrm{Pi}-\mathrm{Oi})^{2}}{\mathrm{n}}} \\
& \mathrm{d}=\left[\frac{\sum_{\mathrm{i}=1}^{\mathrm{n}}(\mathrm{Pi}-\mathrm{Oi})^{2}}{\sum_{\mathrm{i}=1}^{\mathrm{n}}(|\mathrm{Pi}-\overline{\mathrm{O}}|+|\mathrm{Oi}-\overline{\mathrm{O}}|)^{2}}\right]
\end{aligned}
$$

em que Pi corresponde aos valores de razão de umidade preditos pelo modelo (\%), Oi corresponde aos valores de razão de umidade observados/experimentais (\%) e $\mathrm{n}$ corresponde ao número de dados obtidos (adimensional); $\overline{\mathrm{O}}$ corresponde à média dos valores de razão de umidade observados/experimentais (\%).

\section{Resultados e discussão}

Os processos de concentração dos extratos de café realizados em evaporador rotativo (simulação de concentração sob vácuo parcial) e em recipiente inoxidável (simulação de concentração em tacho aberto) apresentaram tempo de duração semelhante, de 2 h 30min e 2 h 40 minutos, respectivamente. Contudo, o extrato concentrado em tacho aberto pode apresentar aroma característico de fumaça, já que o produto pode ficar depositado na superfície, causando superaquecimento e promovendo sabores e odores indesejáveis.

$\mathrm{O}$ agente espumante Emustab $^{\circledR}$ foi utilizado na concentração de $6 \%$ e possibilitou boa estabilidade à espuma formada. De acordo com outros trabalhos (Araújo et al., 2017; Cruz et al., 2017; Guimarães et al., 2017), Emustab ${ }^{\circledR}$ apresentou resultados superiores se comparado com outros agentes espumantes.

Entretanto, o extrato concentrado em tacho aberto apresentou mais dificuldade na formação de espuma se comparado com o extrato concentrado em evaporador rotativo. Esse resultado pode estar relacionado com a provável alteração das propriedades físico-químicas da amostra, em razão da exposição a temperaturas mais altas durante o processo desse tratamento.

O processo de secagem permite a obtenção do café solúvel após a concentração do extrato. Várias metodologias podem ser utilizadas, contudo a utilização da secagem em leito de espuma pode proporcionar uma secagem mais simples e com menor custo (Araújo et al., 2017). Além disso, durante a secagem, alguns parâmetros como temperatura, umidade e velocidade do ar podem ser monitorados a fim de otimizar o processo.

A Figura 1 indica a taxa exponencial da razão de umidade (RU) em função do tempo de secagem para diferentes temperaturas e demonstra que houve perda mais rápida de umidade nas primeiras horas da secagem em todas as temperaturas testadas. Também é possível perceber que quanto maior a temperatura empregada, menor o tempo de secagem, evidenciando que o fator térmico é determinante na velocidade de perda de umidade do produto, devido, principalmente, ao aumento do calor transferido para a amostra, fornecendo mais força-motriz para a evaporação da umidade (Salahi et al., 2017). Tais características também foram observadas em diversos estudos com diferentes alimentos (Araújo et al., 2017; Franco et al., 2016; Roncheti, 2014; Santos et al., 2010). 


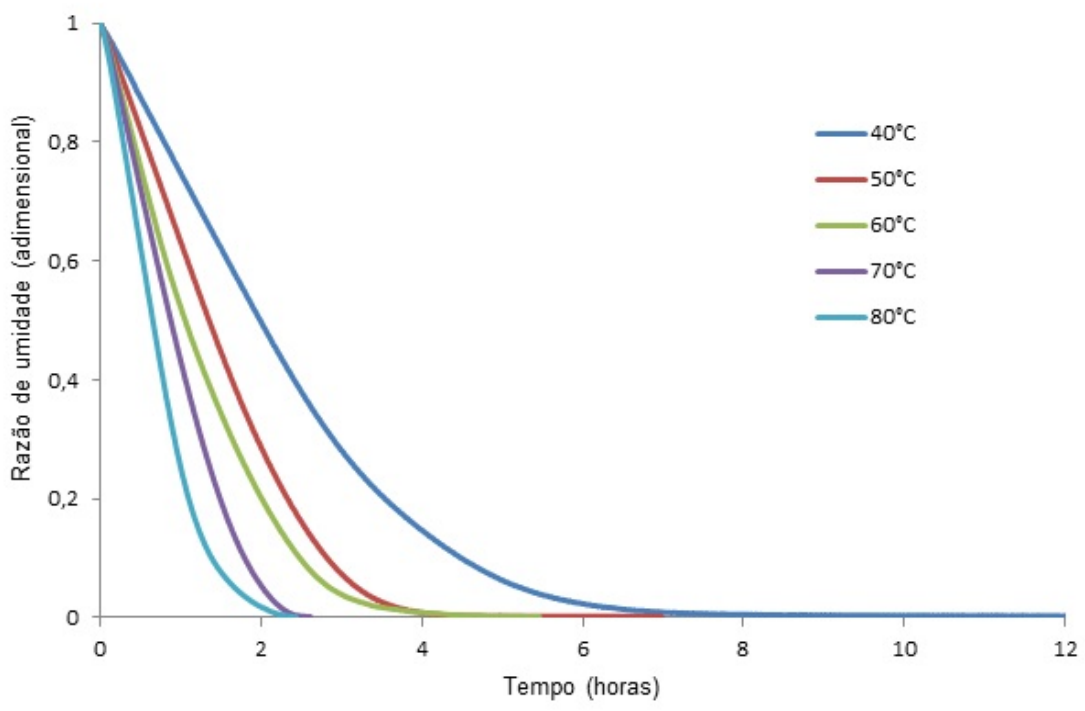

Figura 1. Curvas de secagem de café em leito de espuma em diferentes temperaturas.

O ajuste dos dados ao modelo de Page por meio da análise de regressão não linear resultou nas equações de razão de umidade (RU) em função do tempo (t), com valores de coeficientes de determinação correspondentes, para as respectivas temperaturas de secagem, conforme mostra a Tabela 1.

Tabela 1. Parâmetros dos modelos ajustados e valores de coeficientes de determinação $\left(\mathrm{R}^{2}\right)$.

\begin{tabular}{cll}
\hline Temperatura $\left({ }^{\circ} \mathbf{C}\right)$ & Equação & $\boldsymbol{R}^{2}$ \\
\hline 40 & $\mathrm{RU}=\mathrm{e}^{(-0,2688 \mathrm{t})^{1,4184}}$ & 0,999 \\
\hline 50 & $\mathrm{RU}=\mathrm{e}^{(-0,4554 \mathrm{t})^{1,532}}$ & 0,998 \\
\hline 60 & $\mathrm{RU}=\mathrm{e}^{(-0,6479 t)^{1,3532}}$ & 0,998 \\
\hline 70 & $\mathrm{RU}=\mathrm{e}^{(-0,8818 \mathrm{t})^{1,5646}}$ & 0,997 \\
\hline 80 & $\mathrm{RU}=\mathrm{e}^{(-1,3808 \mathrm{t})^{1,5224}}$ & 0,999 \\
\hline
\end{tabular}

Os valores dos coeficientes de determinação se mostraram excelentes para o ajuste dos dados experimentais ao modelo de Page, conforme evidenciado por Santos et al. (2010), em secagem de carambola em secador de bandeja. A aplicação do teste estatístico t de Student também apresentou parâmetros significativos, confirmando que o modelo de Page se ajusta satisfatoriamente à cinética da secagem do extrato de café em leito de espuma.

Para validar os resultados, foram comparados os valores experimentais e os valores estimados pelo modelo nas diferentes temperaturas testadas $\left(40,50,60,70\right.$ e $\left.80^{\circ} \mathrm{C}\right)$, conforme mostra a Figura 2. 


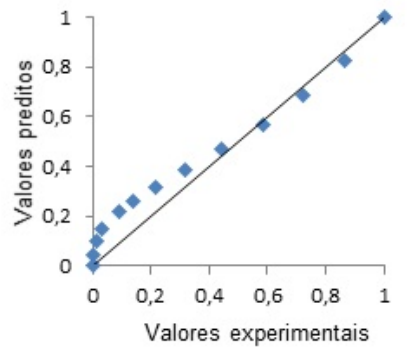

C

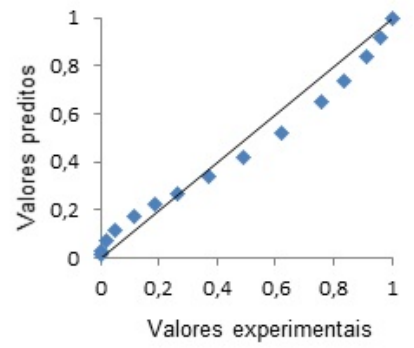

$\mathrm{E}$

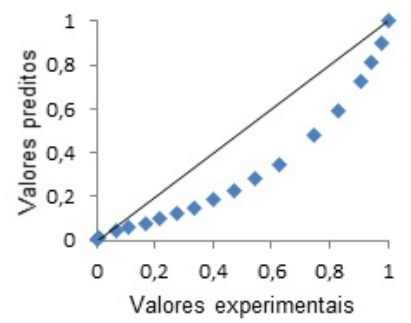

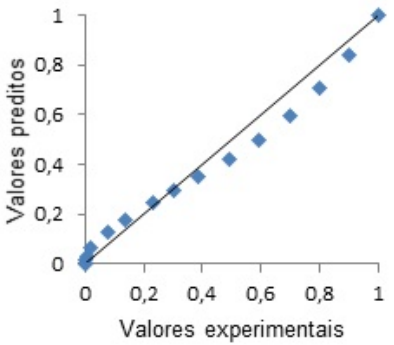

D

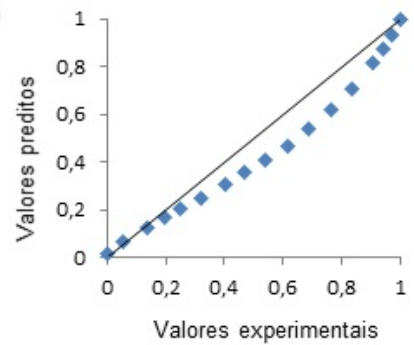

Figura 2. Comparação entre valores de razão de umidades experimentais e preditos, para secagem de café em leito de espuma a $40{ }^{\circ} \mathrm{C}(\mathrm{A}), 50{ }^{\circ} \mathrm{C}(\mathrm{B}), 60^{\circ} \mathrm{C}(\mathrm{C}), 70^{\circ} \mathrm{C}(\mathrm{D})$ e $80{ }^{\circ} \mathrm{C}(\mathrm{E})$.

É possível inferir que há mais tendência de dispersão entre os valores experimentais e preditos na faixa central do gráfico e mais proximidade entre esses valores nas faixas inicial e final da secagem.

A Tabela 2 mostra os resultados dos erros experimentais, permitindo concluir que houve forte correlação entre os dados experimentais e preditos, validando o ajuste ao modelo de Page. Os valores de coeficiente de concordância de Wilmott (d) foram maiores que 0,985 para os tratamentos com 40, 50, 60 e $70{ }^{\circ} \mathrm{C}$, confirmando a exatidão do modelo e a validação dos resultados.

Tabela 2. Erros experimentais obtidos pela validação dos resultados da secagem de café em leito de espuma.

\begin{tabular}{ccccc}
\hline Temperatura $\left({ }^{\circ} \mathbf{C}\right)$ & EAM (\%) & EME (\%) & REQM (\%) & d \\
\hline 40 & 3,6 & $-3,1$ & 5,5 & 0,985 \\
\hline 50 & 1,8 & $-0,2$ & 3,1 & 0,994 \\
\hline 60 & 4,3 & $-0,6$ & 5,3 & 0,989 \\
\hline 70 & 4,0 & 3,0 & 6,3 & 0,986 \\
\hline 80 & 8,4 & 7,9 & 13,0 & 0,942 \\
\hline
\end{tabular}

Erro absoluto médio (EAM), erro médio de estimativa (EME) e raiz do erro quadrático médio (REQM), além do coeficiente de concordância de Wilmott (d).

Entretanto, o processo de secagem utilizando a temperatura de $80{ }^{\circ} \mathrm{C}$ gerou valores de erros mais altos $(\mathrm{EAM}=8,4 \%$; $\mathrm{EME}=7,9 \%$; REQM $=13 \%)$, além de apresentar mais discrepância entre os dados experimentais e preditos $(d=0,942)$. Tais resultados podem ser justificados por uma formação incompleta 
de espuma, que foi percebida visualmente durante o teste experimental, o que pode ter comprometido o desprendimento de água durante a secagem, podendo gerar mais diferenças entre os dados experimentais. Diante disso, comprova-se a importância da produção de uma emulsão permanente para obter mais eficiência quanto à perda de umidade do material a ser seco, aumentando a qualidade (Cruz et al., 2017).

O parâmetro k apresentou crescimento linear com o aumento da temperatura de secagem (Figura 3), que está relacionado com a velocidade de secagem, dependendo diretamente da difusividade efetiva do vapor de água durante a secagem. Quanto ao parâmetro n, não houve relação direta com a temperatura. Uma equação similar também foi proposta por Roncheti (2014), que observou o mesmo comportamento no processo de secagem.

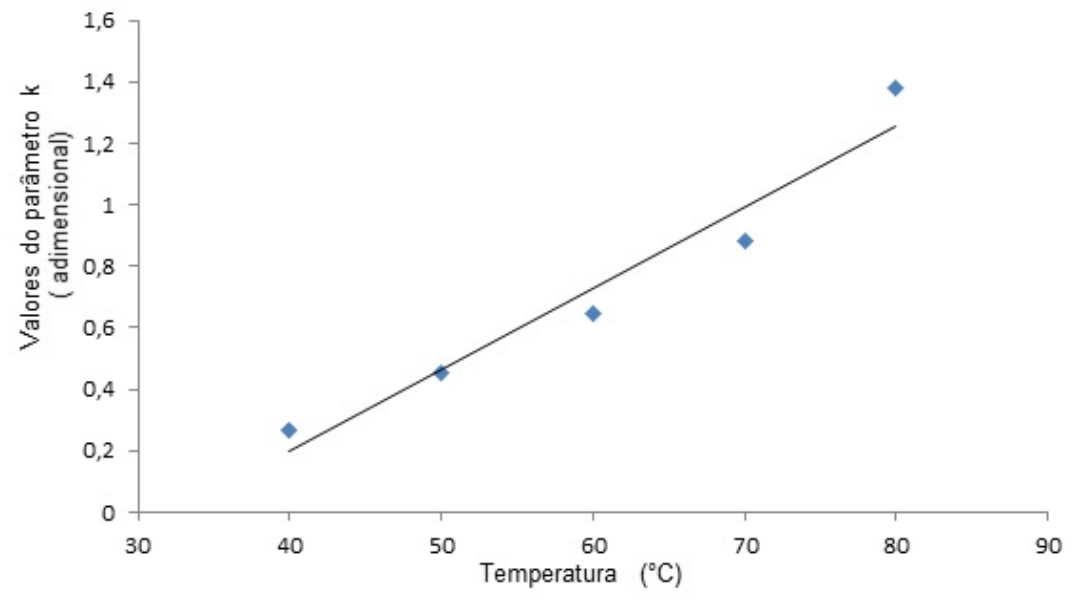

Figura 3. Dados experimentais dos valores do parâmetro k (do modelo de Page) em função da temperatura de secagem.

Assim, foi obtido um modelo capaz de exprimir a razão de umidade (RU) em função da temperatura (T) e do tempo de secagem (t). A Equação 6 representa o modelo multivariado obtido por meio de regressão não linear:

$\mathrm{RU}=\mathrm{e}^{(-(0,0221 \mathrm{~T}-0,6250) \mathrm{t})^{1,4349}}$

em que RU é a razão de umidade, $\mathrm{T}$ é a temperatura e t é o tempo de secagem.

O coeficiente de determinação encontrado foi de 0,995 , evidenciando um ajuste excelente dos dados experimentais ao modelo tridimensional obtido.

Com base na análise da Figura 4, é possível perceber que os dados estão aleatoriamente dispersos e próximos à bissetriz que corta o gráfico, mostrando forte correlação entre os valores experimentais e preditos. Os cálculos dos indicadores estatísticos $(\mathrm{EAM}=1,7 \%$; $\mathrm{EME}=1,5 \%$; REQM $=0,1 \%)$, bem como do coeficiente de concordância de Wilmott $(\mathrm{d}=0,997)$, evidenciam que o modelo representa satisfatoriamente o processo. Além disso, tais indicadores foram menores que os encontrados com as equações individuais por temperatura, indicando capacidade preditiva ainda maior do modelo multivariado proposto. 


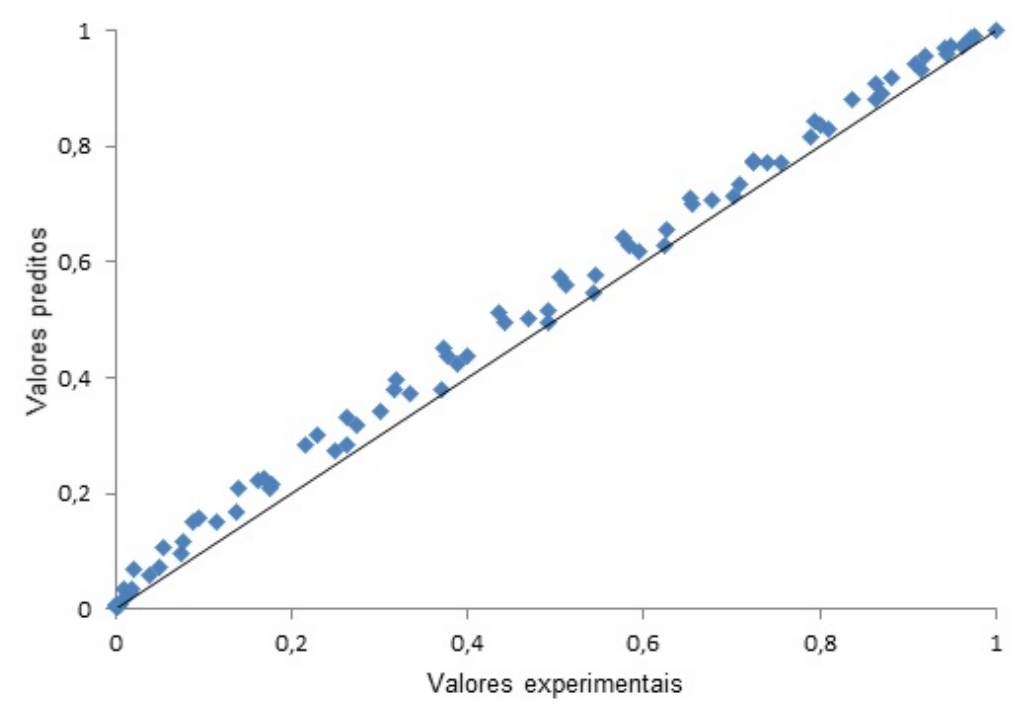

Figura 4. Comparação entre os valores experimentais e preditos pelo Modelo de Page para secagem de café em leito de espuma.

\section{Conclusões}

A utilização do leito de espuma para secagem de extrato concentrado de café mostrou ser uma metodologia eficiente para a obtenção de café solúvel em diferentes temperaturas. Considerando o parâmetro $\mathrm{k}$ do modelo de Page e a razão de umidade de valores preditos e experimentais, a temperatura ótima de secagem pode ser considerada como $50{ }^{\circ} \mathrm{C}$. No entanto, para conhecer a condição ótima de secagem, análises físico-químicas e sensoriais devem ser realizadas, para correlacionar tempo de secagem e qualidade final do produto obtido.

A utilização do modelo de Page foi satisfatória para descrever a cinética do processo de secagem e os resultados foram validados.

Foi verificada uma relação diretamente proporcional entre o parâmetro $\mathrm{k}$, do modelo de Page, e a temperatura de secagem, dando origem a um novo modelo que apresenta a razão de umidade em função do tempo e da temperatura do processo. Tais modelos também poderão ser empregados em dimensionamentos e estimativas de processos para outros alimentos.

\section{Agradecimentos}

Os autores agradecem às agências de fomento: Conselho Nacional de Desenvolvimento Científico e Tecnológico (CNPq), Fundação de Amparo à Pesquisa do Estado de Minas Gerais (FAPEMIG) e à Coordenação de Aperfeiçoamento de Pessoal de Nível Superior (CAPES) pelo financiamento.

\section{Referências}

Araújo, C. S., Macedo, L. L., Vimercati, W. C., Saraiva, S. H., Oliveira, A. N., \& Teixeira, L. J. Q. (2017). Cinética de secagem de acerola em leito de espuma e ajuste de modelos matemáticos. Brazilian Journal of Food Technology, 20, e2016152. http://dx.doi.org/10.1590/1981-6723.15216

Bayindirli, L. (1993). Density and viscosity of grape juice as a function of concentration and temperature. Journal of Food Processing and Preservation, 17(2), 147-151. http://dx.doi.org/10.1111/j.1745-4549.1993.tb00231.x

Buljat, A. M., Jurina, T., Jurinjak Tušek, A., Valinger, D., Gajdoš Kljusurić, J., \& Benković, M. (2019). Applicability of foam mat drying process for production of instant cocoa powder enriched with lavender extract. Food Technology and Biotechnology 57(2), 159-170. PMid:31537965. http://dx.doi.org/10.17113/ftb.57.02.19.6064

Companhia Nacional de Abastecimento - CONAB. (2018). Acompanhamento da safra brasileira: Quarto levantamento: Safra 2018 (Vol. 5, No. 4). Brasília. 
Conselho dos Exportadores de Café do Brasil - CECAFÉ. (2019). Relatório mensal janeiro. São Paulo. Recuperado em 17 de abril de 2019, de http://www.sapc.embrapa.br/arquivos/consorcio/informe_estatistico/CECAFE-Relatorio-Mensal-JANEIRO2019.pdf

Cruz, P. L., Saraiva, S. H., \& Teixeira, L. J. Q. (2017). Otimização do processo de secagem em leito de espuma de extrato de café. Enciclopédia Biosfera, 14(26), 479-492.

Esquivel, P., \& Jiménez, V. M. (2012). Functional properties of coffee and coffee by products. Food Research International, 46(2), 488-496. http://dx.doi.org/10.1016/j.foodres.2011.05.028

Evangelista, S. R., Miguel, M. G. P. C., Cordeiro, C. S., Silva, C. F., Pinheiro, A. C. M., \& Schwan, R. F. (2014). Inoculation of starter cultures in a semi-dry coffee (Coffea arabica) fermentation process. Food Microbiology, 44, 87-95. PMid:25084650. http://dx.doi.org/10.1016/j.fm.2014.05.013

Franco, T. S., Perussello, C. A., Ellendersen, L. N., \& Masson, M. L. (2016). Effects of foam mat drying on physicochemical and microstructural properties of yacon juice powder. Lebensmittel-Wissenschaft + Technologie, 66, 503-513. http://dx.doi.org/10.1016/j.Iwt.2015.11.009

Guimarães, M. K. A., Figueirêdo, R. M. F. D., \& Queiroz, A. J. D. M. (2017). Foam-mat drying kinetics of keitt mango pulp. Revista Caatinga, 30(1), 172-180. http://dx.doi.org/10.1590/1983-21252017v30n119rc

Hardy, Z., \& Jideani, V. A. (2017). Foam-mat drying technology: A review. Critical Reviews in Food Science and Nutrition, 57(12), 2560-2572. PMid:26167878. http://dx.doi.org/10.1080/10408398.2015.1020359

International Coffee Organization - ICO. (2019). Total production by all exporting countries. London. Recuperado em $17 \mathrm{de}$ abril de 2019, de http://www.ico.org/prices/po-production.pdf

Kandasamy, P., Varadharaju, N., Kalemullah, S., \& Maladhi, D. (2014). Optimization of process parameters for foam-mat drying of papaya pulp. Journal of Food Science and Technology, 51(10), 2526-2534. PMid:25328192. http://dx.doi.org/10.1007/s13197-012-0812-y

Martinez, S. J., Bressani, A. P. P., Miguel, M. G. C. P., Dias, D. R., \& Schwan, R. F. (2017). Different inoculation methods for semi-dry processed coffee using yeasts as starter cultures. Food Research International, 102, 333-340. PMid:29195956. http://dx.doi.org/10.1016/j.foodres.2017.09.096

Roncheti, E. F. S. (2014). Estudo do processo de secagem em leito de espuma de cenoura, tomate, beterraba e morango (Dissertação de mestrado). Universidade Federal do Espírito Santo, Alegre.

Salahi, M. R., Mohebbi, M., \& Taghizadeh, M. (2017). Development of cantaloupe (Cucumis melo) pulp powder using foam-mat drying method: Effects of drying conditions on microstructural of mat and physicochemical properties of powder. Drying Technology, 35(15), 1897-1908. http://dx.doi.org/10.1080/07373937.2017.1291518

Santos, C. T., Bonomo, R. F., Chaves, M. A., Fontan, R. C. I., \& Bonomo, P. (2010). Cinética e modelagem da secagem de carambola (Averrhoa carambola) em secador de bandeja. Acta Scientiarum. Technology, 32(3), 309-313.

Santos, R. P., Schwantz, R. M., Fraga, M. L., \& Demuner, J. A. (2018). Management system influence on the increase of soluble coffee production: A case in southeastern Brazil. Brazilian Journal of Development, 4(5), 2180-2199.

Financiamento: Ministério da Ciência, Tecnologia e Inovação/Conselho Nacional de Desenvolvimento Científico e Tecnológico (CNPq); Coordenação de Aperfeiçoamento de Pessoal de Nível Superior (CAPES); Fundação de Amparo à Pesquisa do Estado de Minas Gerais (FAPEMIG). 